\title{
A purely distributed implementation of undervoltage load shedding
}

\author{
Bogdan Otomega, Student, IEEE Mevludin Glavic, Member, IEEE Thierry Van Cutsem, Fellow, IEEE
}

\begin{abstract}
A new design of load shedding against long-term voltage instability is proposed. It uses a set of distributed controllers, each monitoring a transmission voltage and controlling a group of loads. Each controller acts in closed-loop, shedding amounts that vary in magnitude and time according to the evolution of its monitored voltage. The whole system operates without information exchange between controllers, the latter being implicitly coordinated through network voltages. The operation, design and robustness of the proposed scheme are illustrated through a small but realistic example.
\end{abstract}

Index Terms-Voltage stability, system protection scheme, undervoltage load shedding, distributed control

\section{INTRODUCTION}

It is well-known that load shedding is a cost effective countermeasure against voltage instability triggered by large disturbances [1].

The location, timing and magnitude of load shedding are three important aspects of this emergency action, which interact with each other [2].

System Protection Schemes (SPS) against voltage instability can be roughly classified into algorithmic decision-based vs. rule-based.

An algorithmic decision-based SPS could exploit the ability of simulating system evolution faster than real-time, when long-term voltage instability is of concern and the fast quasi steady-state simulation technique is used [2]. Assuming that the disturbance has been identified, the minimal postdisturbance load shedding could be efficiently determined using a method of the type described in [3]. This is, however, an open-loop approach that cannot compensate for inevitable modelling inaccuracies (due mainly to uncertainties in load behaviour) as well as possible component failures.

Research is thus being carried out towards the development of more appropriate algorithms, of which Refs. [4]-[7] are a small sample. In particular, Model Predictive Control could be used to devise a closed-loop scheme. However, there are still serious limitations in the proposed algorithms that need to be addressed before a reliable SPS is obtained: heavy computational burden, combinatorial explosion of candidate control sequences, necessity of knowing the whole system state and hence including the state estimator in the loop, inherent complexity decreasing reliability, etc.

B. Otomega (otomega@montefiore.ulg.ac.be) is with the Dept. of Electrical Engineering and Computer Science (Montefiore Institute) of the University of Liège, Sart Tilman B37, B-4000 Liège, Belgium. M. Glavic (glavic@montefiore.ulg.ac.be) is invited professor and T. Van Cutsem (t.vancutsem@ulg.ac.be) is with the Belgian National Fund for Scientific Research (FNRS) at the same department.

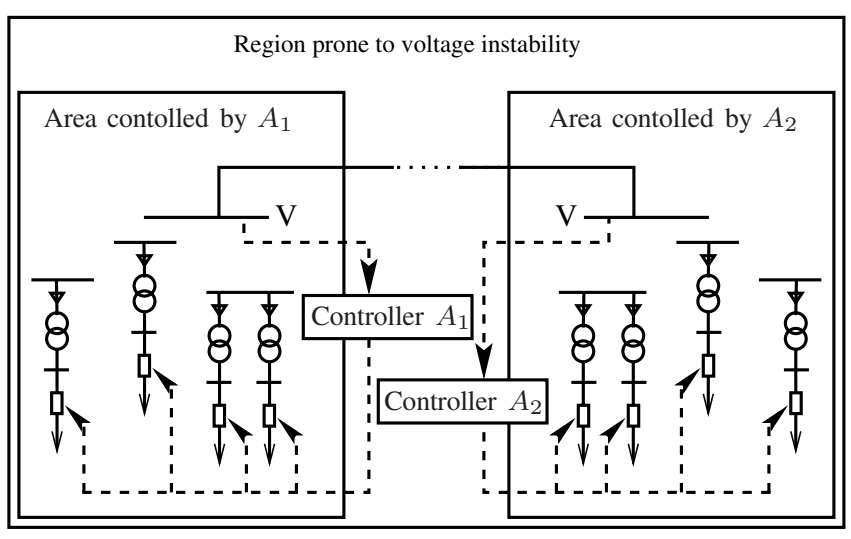

Fig. 1. Overall structure of the proposed scheme

A rule-based SPS relies on simple rules of the type "if voltage drops below some threshold $V^{t h}$ for some duration $\tau$, shed some power $\Delta P$ ". Being much simpler, it is less exposed to failures originating from telecommunications, erroneous models, etc.

Rule-based load shedding usually relies on the detection of low transmission voltages. Clearly, the detection of a low voltage situation is meaningful by itself, as an indication of customer nuisance. Nevertheless, efforts have been paid to develop alternative criteria, having hopefully more anticipation ability. For instance, a voltage instability predictor has been proposed, based on the identification of a Thévenin equivalent from local measurements [8]. However, the underlying assumptions of this technique do not seem compatible with the system dynamics after a large disturbance.

On the other hand, the simplicity of the above rules does not allow the SPS to adjust its action to the severity and location of the disturbance. A step towards better design was made in [9] where the parameters involved in the rules were optimized over a set of scenarios, and an additional rule made the protection operate in closed loop.

The design considered in this paper extends the idea used in [9] by implementing the above mentioned closed-loop rule into several distributed controllers operating in a cooperative way. Furthermore, the scheme proposed here does no longer involve "if ...then ..." rules with fixed time delays and fixed power amounts, and is expected to better adapt to disturbance location.

\section{THE PROPOSED LOAD SHEDDING SCHEME}

The proposed scheme relies on a set of distributed controllers, covering the power system region prone to voltage 
instability. Each controller acts on a set of electrically close loads and monitors the voltage $V$ at a transmission bus located in the same area, as sketched in Fig. 1.

\section{A. Individual controller design}

The decision by a controller to shed load is based on the comparison of $V$ with a threshold value $V^{t h}$. If a (severe) disturbance causes $V$ to become smaller than $V^{\text {th }}$, the controller sheds an amount $\Delta P^{s h}$ of load power after a delay $\tau$. Both $\Delta P^{s h}$ and $\tau$ depend on the dynamic evolution of $V$, as detailed hereafter. Note that the sequence is repeated until the voltage is restored above the threshold.

Let $t_{0}$ be the time where measurement $V$ becomes smaller than $V^{t h}$. A first block of load is shed at a time $t_{0}+\tau$ such that:

$$
\int_{t_{0}}^{t_{0}+\tau}\left(V^{t h}-V(t)\right) d t=C
$$

The $C$ constant has to do with the shedding delay $\tau$. Indeed, the larger $C$, the more time it takes for the integral to reach this value and hence, the slower the action. Furthermore, the control law (1) yields an inverse-time characteristic: the deeper the voltage drops, the less time it takes to reach the value $C$ and, hence, the faster the shedding.

The delay $\tau$ is bounded according to:

$$
\tau_{\min } \leq \tau
$$

to prevent the controller from reacting on a nearby fault (in normal situations time must be left for the protections to act and the voltage to recover to normal values).

The amount of load shed by the controller at $t_{0}+\tau$ is given by:

$$
\Delta P^{s h}=K \cdot \Delta V^{a v}
$$

where $\Delta V^{a v}$ is the average voltage drop over the $\left[t_{0} t_{0}+\tau\right]$ interval, i.e.

$$
\Delta V^{a v}=\frac{1}{\tau} \int_{t_{0}}^{t_{0}+\tau}\left(V^{t h}-V(t)\right) d t
$$

Clearly, the above relationships transpose voltage drop severity into load shedding amplitude: the larger $V^{t h}-V$, the larger $\Delta V^{a v}$ and, hence, the larger the amount of load shed. The same holds true when the gain $K$ increases.

Furthermore, $\Delta P^{s h}$ is bounded as follows:

$$
\Delta P_{\min }^{s h} \leq \Delta P^{s h} \leq \min \left(\Delta P_{\max }^{s h}, P_{\text {int }}(t)\right)
$$

where $\Delta P_{\text {min }}^{s h}$ accounts for the fact that by acting on distribution circuit breakers the controller may not be able to shed small amounts while $\Delta P_{\max }^{s h}$ prevents unacceptable transients caused by large load disconnection, and $P_{\text {int }}(t)$ denotes the part of the load still interruptible at time $t$ (which of course depends on load composition and already disconnected amounts).

Clearly, the reactive power of loads is reduced together with the active power. In the absence of more detailed information, we assume that both powers vary in the same proportion, i.e. the reactive change is given by:

$$
\Delta Q^{s h}=\Delta P^{s h} \frac{P_{i n t}(t)}{Q_{\text {int }}(t)}
$$

where $Q_{\text {int }}(t)$ is the interruptible load reactive power.

At the time the controller sheds load, the integral in $(1,4)$ is reset to zero, $t_{0}$ is set to the current time, and the controller is ready to act again as long as $V<V^{t h}$, and provided that load is available to do so.

It must be emphasized that this repeated action capability yields a closed-loop behaviour, in the sense explained in the Introduction (new actions being decided from response to previous actions). This closed-loop structure is important as it guarantees robustness against operation failures and uncertainties affecting the load behaviour.

\section{B. Cooperation between controllers}

The various controllers interact in the following way.

When the controller monitoring bus $i$ sheds some load, this causes not only the voltage $V_{i}$ at this bus to increase but also the voltages at neighbouring buses monitored by other controllers. Let $j$ be such a bus and $A_{j}$ the corresponding controller $(j \neq i)$. Since $V_{j}$ increases, the integral $\int\left(V^{t h}-V_{j}(t)\right) d t$ grows more slowly with time, thereby leading to a larger delay $\tau$ before $A_{j}$ can act. For the same reason, $\Delta V^{a v}$ decreases and $A_{j}$ will shed less load once the $\tau$ delay is elapsed. Furthermore, if the voltage increase is such that $V_{j}$ becomes larger than $V^{t h}$, controller $A_{j}$ will be reset. In other words, when one controller sheds load, this inhibits or slows down the controllers that compete with him to restore voltages in the same area. This cooperation avoids excessive load shedding.

Furthermore, for already mentioned reasons, there will be a trend to shed first where voltages drop the most. This place changes from one disturbance to another. Hence, the control scheme automatically adjusts the shedding location to the disturbance it faces.

Note that the above features are achieved without resorting to a dedicated communication network. The controllers do not exchange information, but are rather informed of their respective actions through the power system itself. This is made possible by the fact that voltages have no "inertia": the effects of shedding are felt almost instantaneously. Neither do the controllers require a model of the system. This and the absence of communication makes the protection scheme definitely simpler and hence more reliable.

\section{Analogy with multi-agent systems}

The proposed scheme shows a strong analogy with MultiAgent Systems (MAS). MAS have received much attention in various engineering disciplines (e.g. [10]), including power system engineering [11], [12]. In fact, there is no general consensus on what an agent is [10]-[13]. Its meaning is strongly biased by the background field (engineering, artificial intelligence, cognitive science, computer science, software engineering, etc.), although the perception in software engineering and artificial intelligence tends to prevail [11]. Similarly, there is no clear borderline between distributed computing and multi-agent technology.

Nevertheless, in the spirit of the general definitions given in [10], [13], the following analogy between the load shedding controllers and MAS can be made. 
Each controller possesses two distinguishing characteristics of an agent: autonomy (it operates without direct intervention of another controller or supervisor) and some degree of intelligence (in the form of "if ...then ..." rules and Eqs. (1-5)). The proposed scheme can be seen as a MAS, which is a loosely coupled network of problem-solving entities that work together to find answers to problems that are beyond the individual capabilities of each entity [10].

MAS fall into two basic categories: independent and cooperative.

In independent MAS, the individual agents pursue their own goals independently of the others. They can be further classified into MAS with discrete agents, if the goals of the agents bear no relation to one another, or MAS with emergent cooperation, if agents cooperate "with no intention of doing so" and cooperation emerges from the consistency of their individual goals. An example of the latter is the combination of secondary voltage control and undervoltage load shedding.

On the other hand, cooperative agents have the same goals, domain knowledge and possible actions. The only difference among individual agents is their sensory inputs, i.e. they are situated at different places in the environment. Cooperative agents can be further classified into communicating and noncommunicating. In the former case, they usually rely on a dedicated communication system.

The proposed load shedding scheme can be categorized as a cooperative non-communicating MAS. Indeed, in the proposed scheme, there is an implicit communication and coordination between agents, through system voltages, as explained in the previous section.

\section{Tuning the control scheme}

The tuning of the controllers mainly consists in choosing the best possible values for the $C, K$ and $V^{\text {th }}$ parameters. The bounds $\tau_{\min }, \Delta P_{\min }^{s h}$ and $\Delta P_{\max }^{s h}$ can be chosen by engineering judgement.

Using the same $C, K$ and $V^{t h}$ values for all controllers makes the design definitely simpler. In the tests we performed so far, there has been no evidence that individual values would yield substantial benefits.

The tuning of these parameters should be such that [9]:

- no load is shed in stable cases

- all unstable cases are saved

- as few load as possible is shed when needed

- the nuisance of low voltages on customers is minimized.

In this respect, $V^{\text {th }}$ should be low enough in order the protection not to act in acceptable post-disturbance situations (typically for $\mathrm{N}-1$ contingencies) but high enough to prevent load voltages from reaching unacceptable values.

Further illustrations of the above points are provided in the next section.

\section{Simulation Results}

\section{A. Test system and control scheme}

The proposed scheme has been tested on the Nordic32 test system used by a CIGRE Task Force on Long-term

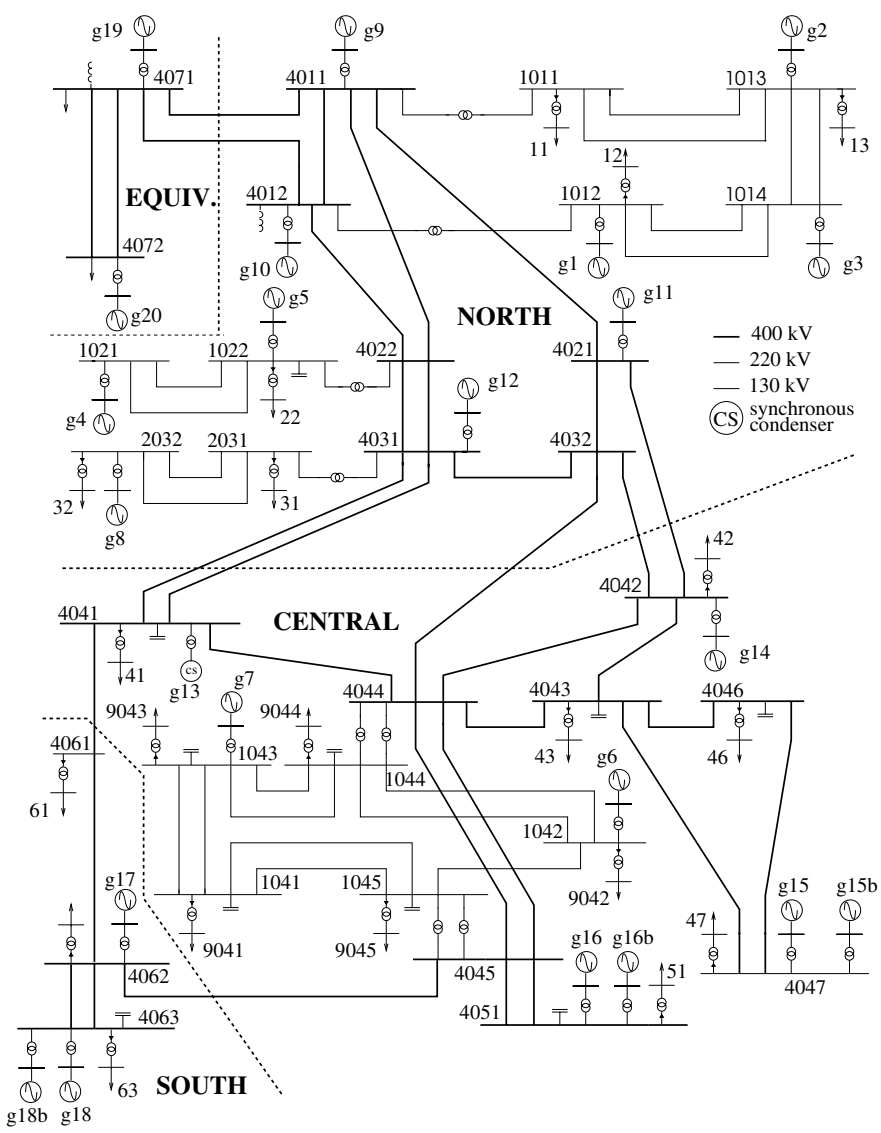

Fig. 2. Nordic32 test system one-line diagram

Dynamics. The data can be found in [14] while the one-line diagram is shown in Fig. 2. The model includes 55 buses, 23 generators and 22 voltage-sensitive loads. The long-term dynamics is driven by transformer load tap changers and generator overexcitation limiters.

Voltage problems are experienced in the "Central" region (see Fig. 2). This area has been thus provided with five controllers, as detailed in Table I. In this simple system, each controller monitors the voltage of one transmission bus and controls the load on the distribution side of the transformer connected to that bus. In a real-life application, however, each controller would act on a set of loads located at different buses, as sketched in Fig. 1.

TABLE I

CONSIDERED CONTROLLERS

\begin{tabular}{|c|c|c|c|}
\hline $\begin{array}{c}\text { Controller } \\
\text { name }\end{array}$ & $\begin{array}{c}\text { Monitored } \\
\text { bus }\end{array}$ & $\begin{array}{c}\text { Controlled } \\
\text { load bus }\end{array}$ & $\begin{array}{c}\text { Available power } \\
\text { to shed (MW) }\end{array}$ \\
\hline$A_{1041}$ & 1041 & 9041 & 600 \\
\hline$A_{1042}$ & 1042 & 9042 & 300 \\
\hline$A_{1043}$ & 1043 & 9043 & 230 \\
\hline$A_{1044}$ & 1044 & 9044 & 800 \\
\hline$A_{1045}$ & 1045 & 9045 & 700 \\
\hline
\end{tabular}

\section{B. Detailed example of performance}

We consider hereafter a double contingency which consists of tripping line 4032-4044 and generator g14 (see Fig. 2). The unstable system response experienced without load shedding is shown with dotted line in Fig. 3, showing the evolution of 
voltage at bus 1041 provided by quasi steady-state simulation. The heavy line in the same figure corresponds to the system stabilized by the proposed control scheme.

The parameters given in Table II are used for all controllers and were chosen in conformity with the results presented in Section III-G. With the shown values of $V^{t h}, C$ and $K$, if the voltage settles at $0.87 \mathrm{pu}$, for instance, $80 \mathrm{MW}$ are shed after 15 seconds. Also, the lower bound of 3 seconds on the delay starts being enforced for a voltage drop equal to $0.10 \mathrm{pu}$; the power shed is then $400 \mathrm{MW}$. For larger voltage drops, the delay remains 3 seconds, but more power is shed.

TABLE II

CONTROLLER SETTINGS FOR THE EXAMPLE OF FIgS. 3 TO 5

\begin{tabular}{|c|c|}
\hline$V^{t h}$ & $0.89 \mathrm{pu}$ \\
$C$ & $0.3 \mathrm{pu} \cdot \mathrm{s}$ \\
$K$ & $4000 \mathrm{MW} / \mathrm{pu}$ \\
$\tau_{\min }$ & $3 \mathrm{~s}$ \\
$\Delta P_{\min }^{s h}$ & $10 \mathrm{MW}$ \\
\hline
\end{tabular}

In this example, controllers $A_{1041}$ and $A_{1044}$ responded to the disturbance. In order to illustrate their interactions, a zoom of the dashed area of Fig. 3 is given in Fig. 4, while Fig. 5 shows the voltage monitored by controller $A_{1044}$ over the same time interval. In both figures, the MW values refer to the power shed by the controller of concern while the circles indicate shedding by the other one.

As can be seen, the $64 \mathrm{MW}$ shed by $A_{1041}$ make the voltage at bus 1044 recover above $V^{\text {th }}$, with the effect of resetting $A_{1044}$. Similarly, the voltage jump experienced when $A_{1041}$ sheds 72 MW delays and reduces the first load shedding by $A_{1044}$.

Figure 5 also illustrates the previously mentioned inversetime characteristic. The two hatched areas have the same surface $C$. Since the voltage is lower after the first shedding than after the third one, the controller waits less before the second shedding than before the fourth one.

\section{Sensitivity to parameters $V^{\text {th }}, C$ and $K$}

Figure 6 illustrates the control scheme performance for various settings $V^{\text {th }}, C$ and $K$.

The stars indicate settings for which the post-disturbance evolution is accepted. The criterion is that all transmission voltages remain above $0.85 \mathrm{pu}$. This value, smaller than $V^{t h}$, corresponds to unacceptable customer voltages as well as a high risk of field-current limited generators to lose synchronism.

The dots indicate failures, i.e. cases where the $0.85 \mathrm{pu}$ voltage was temporarily or permanently crossed. Expectedly, the lower $V^{\text {th }}$, the less time the controllers have to prevent voltages from reaching $0.85 \mathrm{pu}$, and hence a smaller $C$ has to be chosen. This can also be compensated by a larger $K$.

\section{Amount of load shedding}

The plot of Fig. 7 shows the total power shed (by all controllers at all times), for various values of $C$ and $K$. The white parts correspond to protection failures, according to

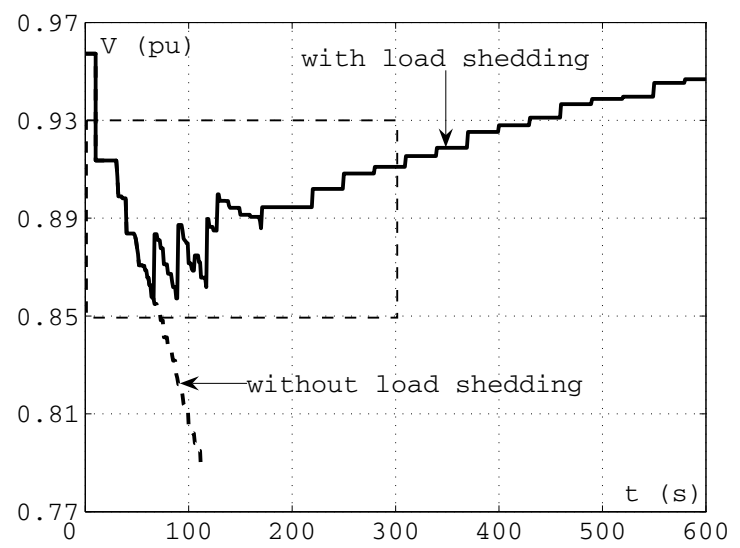

Fig. 3. Voltage evolution at bus 1041 without and with load shedding

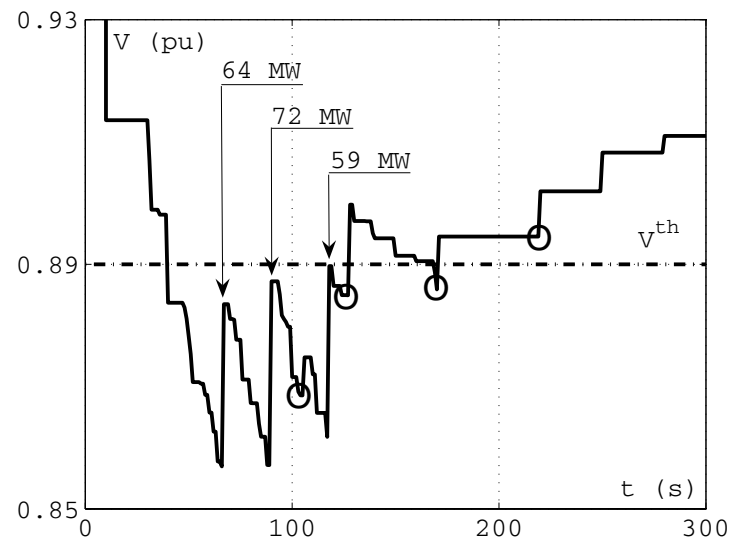

Fig. 4. Monitored voltage and actions of controller $A_{1041}$

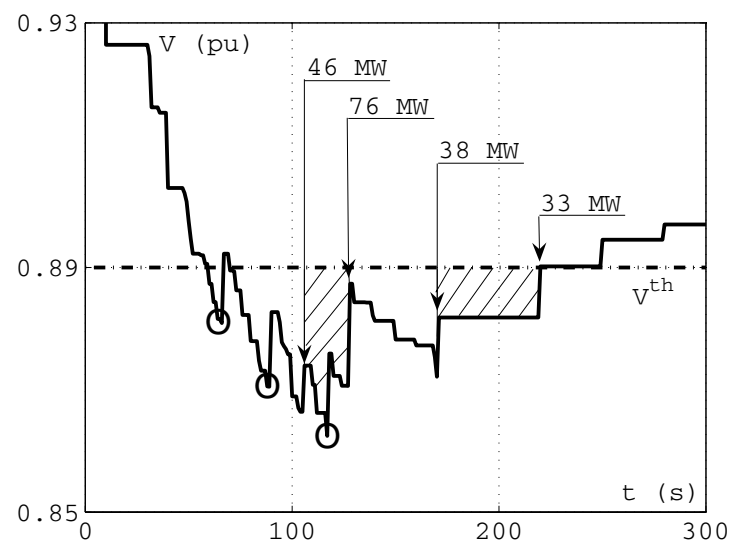

Fig. 5. Monitored voltage and actions of controller $A_{1044}$

the criterion defined in the previous subsection. The settings should correspond to the darkest points, if possible.

The figure confirms that choosing a larger $C$ (which means a slow responding protection) requires to also set $K$ to a larger value, but leads to shedding more load. Beyond some value of $C$, the protection is so slow that it fails, whatever the value of $K$.

Note that the zones of equal shedding are not limited by smooth boundaries. This is attributable to the discrete nature of the controllers, already explained in Section II-C. For instance, with reference to Figs. 4 and 5, a smooth change of a parameter 


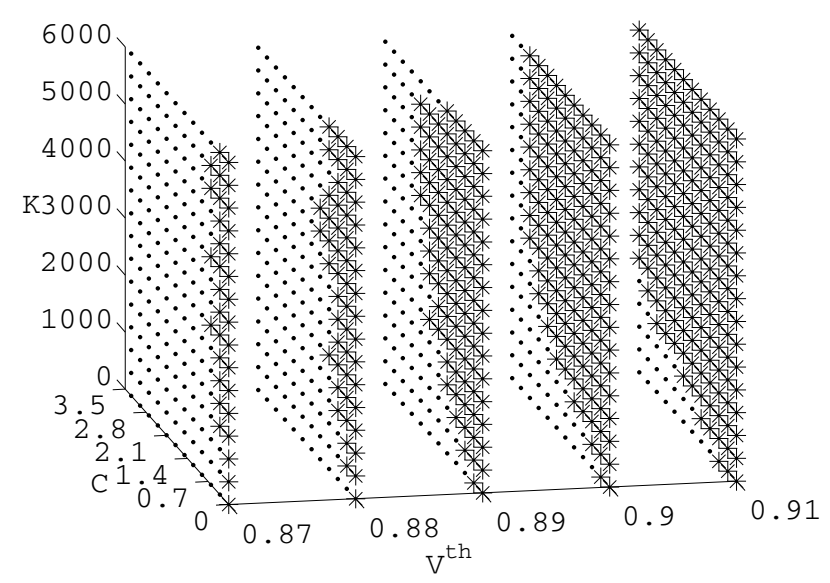

Fig. 6. Performance of load shedding scheme for different settings

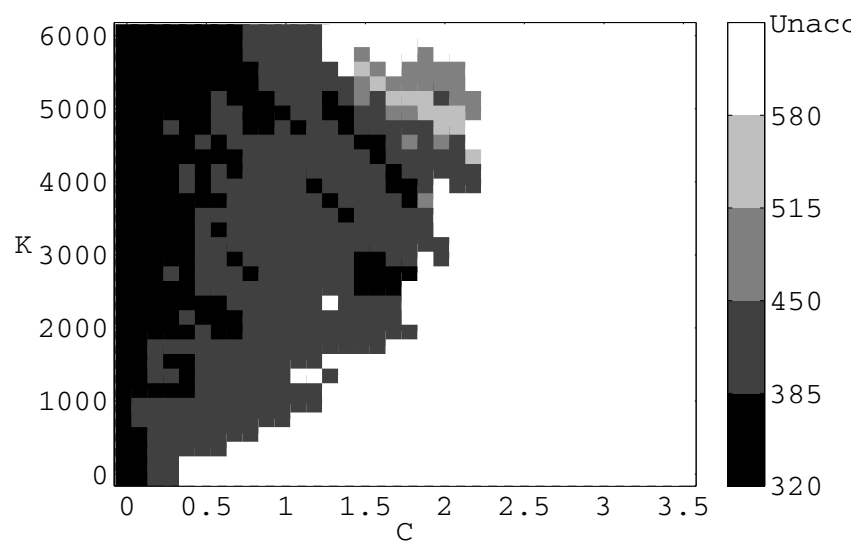

Fig. 7. Total power shed for various $(C, K)$ values, with $V^{t h}=0.89 \mathrm{pu}$

may lead to a smaller first shedding by controller $A_{1041}$, so that $A_{1044}$ does not reset but is only delayed, and the sequence of actions changes.

One can notice that in Fig. 7, for $C=0$, all values of $K$ lead to almost the same amount of load shedding. This is explained as follows. For $C=0$ the delay is lower limited to $\tau_{\min }=3 \mathrm{~s}$; the protection will thus act many times and voltages will recover fast. In this case, it does not really matter whether the protection sheds 35 blocks of 10 MW (corresponding to $K \simeq 500$ ) or 10 blocks of $35 \mathrm{MW}$ (corresponding to $K \simeq 1750$ ): in both cases, shedding is taking place fast enough so that it is not required to shed more to compensate for shedding more slowly [2], [9].

Note, however, that using too small $C$ values is not recommended because the integral in Eq. (4) is computed over a short interval where transients may deteriorate accuracy. Using too small $K$ values is not recommended either because it may not be feasible to disconnect small blocks of loads.

\section{E. Robustness of the control scheme}

In order to illustrate the robustness of the proposed design, Table III shows the power shed by each controller in various scenarios. Case 1 corresponds to the simulation shown in Figs. 3 to 5 while the other cases correspond to failures, as detailed hereafter.
In Case 2 it is assumed that only $20 \%$ of the load are interruptible at bus 1041. This is compensated by a stronger action of $A_{1044}$ and an intervention of $A_{1043}$.

In Case 3 we suppose that the voltage measurement used by $A_{1041}$ is $0.01 \mathrm{pu}$ smaller than the correct value, causing this controller to act faster and shed more power. This is compensated by a smaller action of $A_{1044}$.

Case 4 simulates a full failure of $A_{1041}$; this is covered by a stronger action of $A_{1043}$ and $A_{1044}$. Similarly, Case 5 corresponds to failure of both $A_{1041}$ and $A_{1044}$, leading $A_{1042}$ and $A_{1045}$ to come into play.

Clearly, this redundancy among controllers makes the protection scheme very reliable. In this example, the total power shed even decreases as more controllers compensate for those that should have responded first to the voltage drops.

TABLE III

LOAD SHEDDING AMOUNT (MW) IN VARIOUS SCENARIOS

\begin{tabular}{|c||c|c|c|c|c|}
\hline \multicolumn{1}{|c||}{ Controller } & \multicolumn{5}{c|}{ Case } \\
& 1 & 2 & 3 & 4 & 5 \\
\hline$A_{1041}$ & 195 & 120 & 206 & - & - \\
\hline$A_{1042}$ & 0 & 0 & 0 & 0 & 102 \\
\hline$A_{1043}$ & 0 & 39 & 0 & 120 & 229 \\
\hline$A_{1044}$ & 193 & 220 & 159 & 244 & - \\
\hline$A_{1045}$ & 0 & 0 & 0 & 0 & 33 \\
\hline \hline Total & 388 & 379 & 365 & 364 & 363 \\
\hline
\end{tabular}

\section{F. Comparison with centralized load shedding}

The distributed scheme has been also compared with a centralized design in which a single controller of the type described in Section II-A acts on all loads. More precisely, the input signal used is the average of the five bus voltages listed in Table I, while shedding is distributed homothetically over the five loads in proportion to their pre-disturbance powers.

The corresponding results are given in Fig. 8, that should be compared to Fig. 7 .

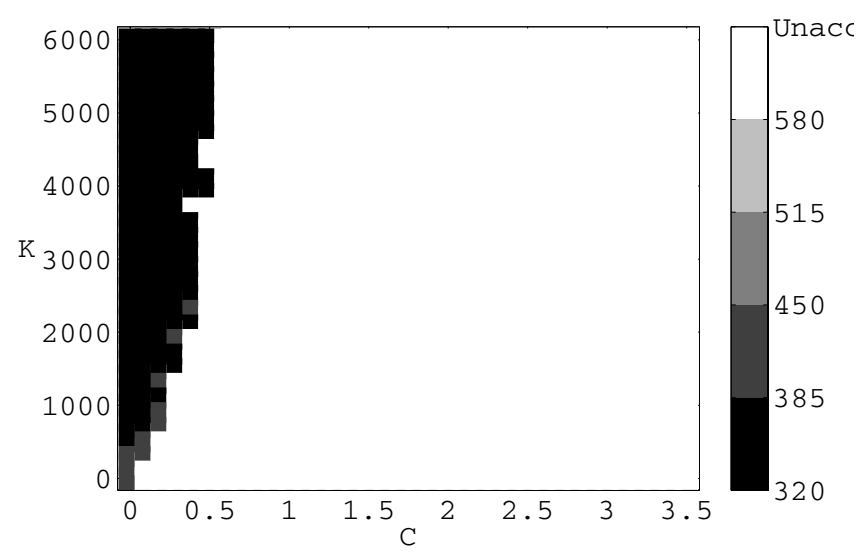

Fig. 8. Total power shed for various $(C, K)$ values $\left(V^{t h}=0.89 \mathrm{pu}\right)$

The performance is similar in terms of amount of load shedding. However, the range of admissible $(C, K)$ values is much smaller. This leaves a reduced security margin with respect to protection failure, even more since small values of $C$ are not recommended, as explained above. Furthermore, this scheme does not offer the redundancy of the distributed design 
and individual voltages are less well controlled. Finally, worse results are to be expected with this controller when applied to a larger system.

\section{G. Choosing a design for various contingencies}

An undervoltage load shedding scheme must be designed to operate in a wide range of scenarios, characterized by various operating conditions and disturbances. The optimization of an undervoltage protection over a set of scenarios was considered in [9]. A similar procedure can be followed for the proposed scheme, as detailed hereafter.

First, "mild" disturbances for which no shedding is needed should be considered. The protection should be tuned so that no load is shed. This can be done by setting the voltage threshold $V^{\text {th }}$ low enough.

Second, for severe disturbances requiring load shedding, plots of the type shown in Fig. 7 can be determined. From these, a final common value of $(C, K)$ can be chosen either by engineering judgement or by minimizing the total load shedding as in [9].

An illustrative example follows.

We consider all N-1 (line or generator tripping) contingencies which satisfy the criterion mentioned in Section III-C. Note that, according to standard practice, all N-1 contingencies should be involved; however, the Nordic32 test system is very stressed and cannot withstand the loss of generator g6, g14, $\mathrm{g} 15, \mathrm{~g} 15 \mathrm{~b}$ and $\mathrm{g} 16$, which are thus not included in the list (i.e. load shedding will be allowed for these five contingencies). The lowest voltages reached at the five buses monitored by controllers, following any of the above contingencies, are given in Table IV. One can see that by setting $V^{\text {th }}$ to $0.89 \mathrm{pu}$, no controller will be triggered and hence, no load will be unduly shed. This threshold is thus selected for the remaining of the procedure.

TABLE IV

LOWEST VOLTAGES AFTER N-1 CONTINGENCIES

\begin{tabular}{|c|c|}
\hline bus & min. volt. (pu) \\
\hline 1041 & 0.90 \\
\hline 1042 & 0.98 \\
\hline 1043 & 0.93 \\
\hline 1044 & 0.94 \\
\hline 1045 & 0.95 \\
\hline
\end{tabular}

Next, we consider the following four severe contingencies requiring load shedding (more disturbances should be considered in practice, but are not included here due to space limitations):

C1: loss of line 4032-4044 and generator g14

C2: loss of double-circuit line 4031-4041

C3: loss of lines 4032-4044 and 4041-4044

C4: loss of lines 4042-4043 and 4042-4044

Figure 9 shows the overall performance of the protection while Figs. 10 to 13 show the variation of the total power shed with $C$ and $K$, for each contingency. From these plots, and from previous considerations, the combination $(C=0.3 \mathrm{pu} \cdot \mathrm{s}$, $K=3000 \mathrm{MW} / \mathrm{pu}$ ) appears to be a reasonable choice.

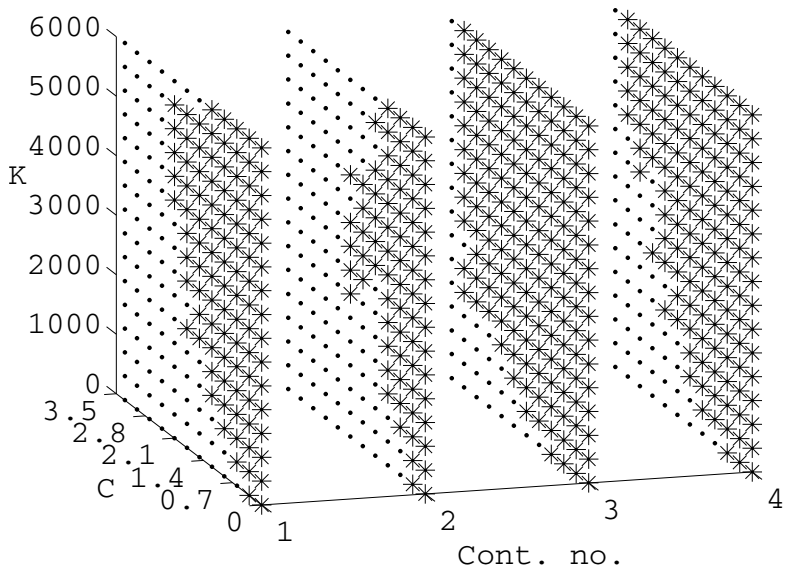

Fig. 9. Performance of load shedding scheme for different contingencies

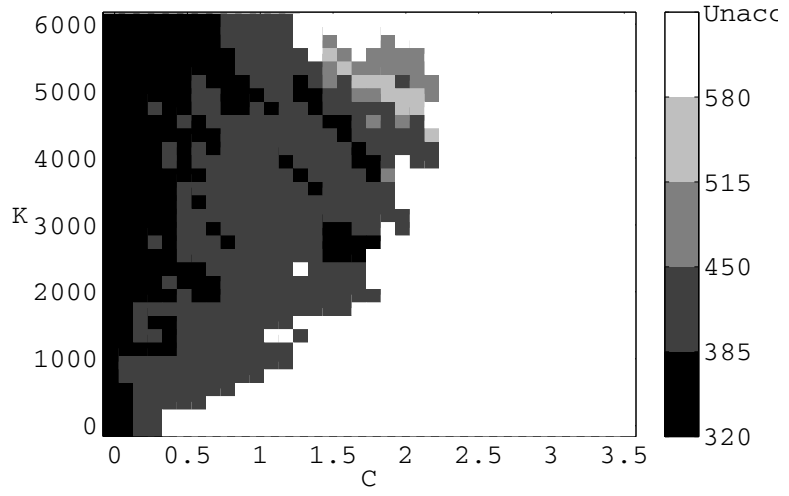

Fig. 10. Total power shed (MW) for various $(C, K)$ values; contingency $\mathrm{Cl}$

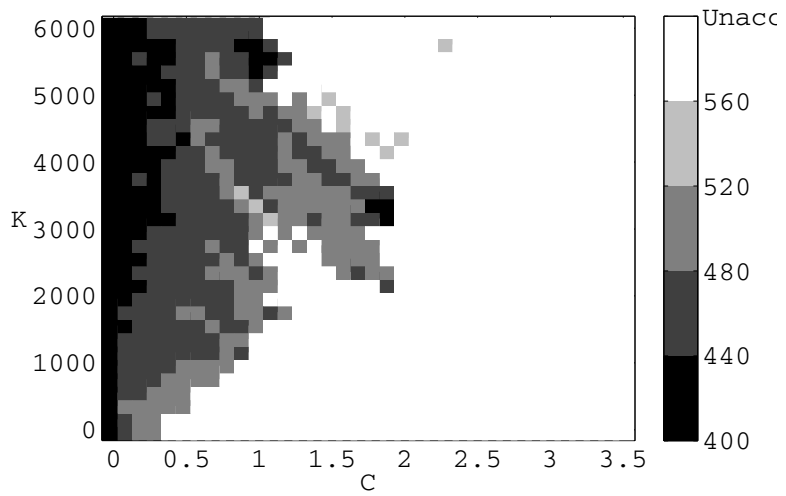

Fig. 11. Total power shed (MW) for various $(C, K)$ values; contingency $\mathrm{C} 2$

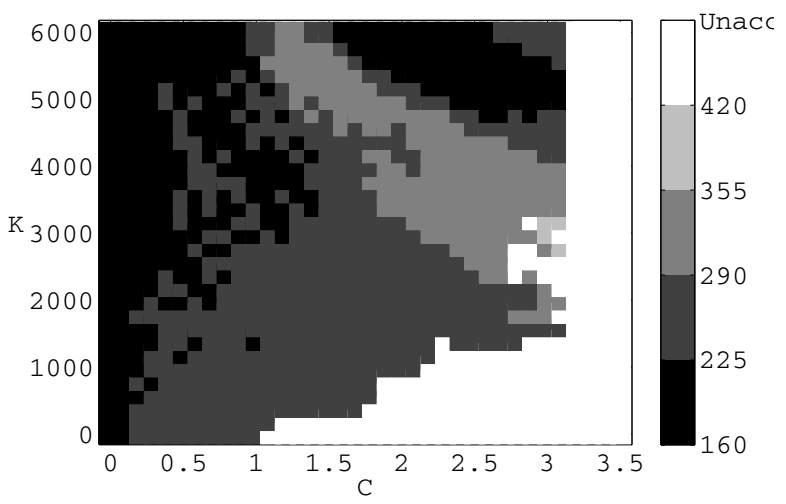

Fig. 12. Total power shed (MW) for various $(C, K)$ values; contingency $\mathrm{C} 3$ 


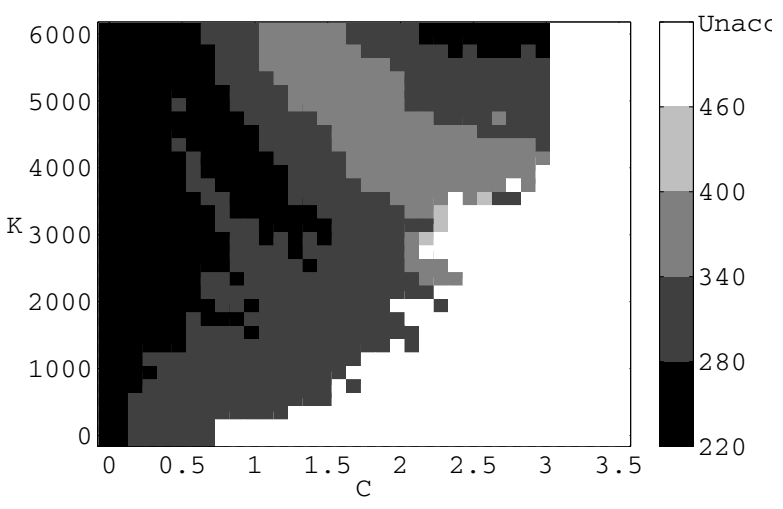

Fig. 13. Total power shed (MW) for various $(C, K)$ values; contingency $\mathrm{C} 4$

\section{CONCLUSION}

This paper outlines a purely decentralized implementation of undervoltage load shedding. The controllers operate in closed-loop, thereby adjusting their emergency action to the severity of the disturbance. Redundancy guarantees robustness against system behaviour uncertainties and operation failures. The controllers are coordinated through the power system itself, without resorting to a dedicated communication network, which adds to simplicity and hence reliability.

The proposed scheme has been successfully tested on the small Nordic32 test system. However, its ability to adjust to the disturbance location has to be demonstrated on a larger system. As of writing this paper, promising results have been obtained on the model of a real-life system including other voltage controls.

\section{REFERENCES}

[1] C. W. Taylor, "Concepts of undervoltage load shedding for voltage stability", IEEE Trans. on Power Delivery, Vol. 7, pp. 480-488, 1992

[2] T. Van Cutsem, C. Vournas, Voltage Stability of Electric Power Systems, Boston, Kluwer Academic Publishers (now Springer), 1998

[3] C. Moors, T. Van Cutsem, "Determination of optimal load shedding against voltage instability", Proc. 13th PSCC conference, Trondheim (Norway), 1999, pp. 993-1000

[4] M. Larsson, D. Karlsson, "Coordinated system protection scheme against svoltage collapse using heuristic search and predictive control", IEEE Trans. Power Systems, vol. 18, pp. 1001-1006, Aug. 2003

[5] M. Zima, G. Andersson, "Stability assessment and emergency control method using trajectory sensitivities", Proc. IEEE PowerTech conference, Bologna (Italy), June 2003

[6] J. Y. Wen, Q. H. Wu, D. R. Turner, C. J. Cheng, J. Fitch, "Optimal coordinated voltage control for power system voltage stability", IEEE Trans. Power Systems, vol. 19, pp. 1115-1122, May 2004

[7] I. A. Hiskens, B. Gong, "Voltage stability enhancement via model predictive control of load", Intelligent Automation and Soft Computing, vol. 12, no. 1, pp. 117-124, 2006

[8] M. Begovic, K. Vu, D. Novosel and M.M. Saha, "Use of local measurements to estimate voltage-stability margin", IEEE Trans. on Power Systems, vol. 14, pp. 1029-1035, Aug. 1999

[9] D. Lefebvre, C. Moors, T. Van Cutsem, 'Design of an undervoltage load shedding scheme for the Hydro-Québec system", Proc. IEEE PES General Meeting, Toronto (Canada), July 13-17, 2003

[10] P. Stone, M. Veloso, "Multi-agent systems: a survey from a machine learning perspective", Autonomous Robots, vol. 8, No 3, pp. 345-383, 2000

[11] C. Rehtanz (editor), Autonomous Systems and and intelligent agents in power system control and operation, Springer Verlag, 2003

[12] A. L. Dimeas, N. Hatziargyriou, "Operation of a Multiagent System for Microgrid Control", IEEE Trans. on Power Systems, Vol. 20, No. 3, pp. 1447-1455, Aug. 2005

[13] S. Russel, P. Norvig, Artificial intelligence - A modern approach, Prentice Hall, 1995.

[14] CIGRE TF 38.02.08 (M. Stubbe, convenor), "Long-term dynamics Phase II", Final report, January 1995 